\title{
Prevalence of Cardiac Arrhythmias among Chronic Obstructive Pulmonary Disease Patients Admitted to Jimma University Medical Center
}

\author{
Wondu Reta Demissie* \\ Department of Biomedical science, Ethiopia
}

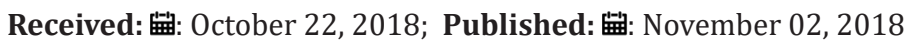

*Corresponding author: Wondu Reta Demissie, Department of Biomedical science, Ethiopia

\begin{abstract}
Background: Cardiac arrhythmias are common in COPD patients and are a major cause of morbidity and mortality.

Aim and Objective: The present study aimed to determine the prevalence of cardiac arrhythmias among patients with COPD

Materials and Methods: The study was conducted on COPD patients visiting chest clinic of Jimma University Medical Center (JUMC) located at Jimma town, South west Ethiopia; from May 18 to August 18, 2017 G.C. A hospital based cross-sectional study was conducted among 80 sampled COPD patients; and an investigation for 12 Lead resting supine ECG was performed. The results of ECG patterns and other variables were entered into EPI data (3.1) and exported to SPSS (20) for further analysis.

Results: The prevalence of arrhythmia accounted for $50 \%$ and the magnitude of its types were classified as Sinus origin arrhythmia (30\%) specifically [Sinus bradycardia (16.3\%), Sinus tachycardia (8.8\%) and Sinus arrhythmia (5.0\%)], Ectopic arrhythmia (20\%) specifically [Premature ventricular Contraction (7.5\%), Atrial fibrillation (6.3\%), Premature atrial contraction (3.8\%), Atrial flutter (1.3\%) and Multi focal atrial tachycardia (1.3\%)], Conduction block arrhythmia (23.8\%) specifically[Bundle branch block (17.5\%) for instance: Complete right bundle branch block (3.8\%), Complete left bundle branch block (5\%), Incomplete right bundle branch block (7.5\%), Incomplete left bundle branch block (1.3\%), Hemi fasicular block (5\%)] and Atrioventricular block (1.3\%)], and Other arrhythmia (11.4\%) like Prolonged QTc interval (8.8\%) and Preexcitation syndrome or Wolf Parkinson white syndrome (2.5\%) as a single COPD patient presented with more than one arrhythmias.
\end{abstract}

Conclusion: Routine ECG investigation should be performed at the setup to screen and initiate early management of Cardio vascular diseases including cardiac arrhythmias for better prognosis COPD patients which was inevitable and very common.

Keywords: Prevalence; Cardiac arrhythmia; Minnesota ECG criteria; COPD

Abbreviations: COPD: Chronic Obstructive Pulmonary Disease; COLD: Chronic Obstructive Lung Disease; CVDs: Cardiovascular Disorders; AF: Atrial Fibrillation; FVC: Forced Vital Capacity

\section{Introduction}

Chronic Obstructive Pulmonary Disease (COPD) is a chronic preventable and treatable multi-system disease where pulmonary component is characterized by significant none fully reversible progressive airflow limitation, often associated with an abnormal inflammatory response of the lung to noxious particles or gases. The best known and widely accepted definition is promulgated by global initiative for chronic obstructive lung disease (COLD), with a post bronchodilator cut-off point of FEV1/FVC ratio <70 [1]. COPD is a leading cause of mortality and morbidity, as WHO estimating it is expected to be third leading cause of death and disability by the year 2020 which has been sixth and fourth in 1990 and 2000 respectively [2]. The prevalence in Africa, though low due to problems of screening like scarce of spirometry, it is expected to rise as a result of an increasingly ageing population and increasing prevalence of the habit of cigarette smoking and exposure to biomass fuel [3].

Heart is the most targetable organ for COPD as systemic complication and develops much pathology of cardiovascular disorders (CVDs) or cardiovascular complications, among which cardiac arrhythmia is the commonest but also other CVDs (angina, 
hypertension, coronary artery disease and congestive health failure) by sharing different risk factors (advanced age, smoking, environmental pollutants, gender, and socioeconomic status) [49]. COPD increases the risk of cardiac arrhythmias and they tend to occur with increasing frequency especially in situations of acute exacerbation, respiratory failure and increasing comorbidities and also vice versa where COPD is highly prevalent among CVDs (about 22.6\%) and in cardiac arrhythmia patients especially in Atrial fibrillation (AF) patients (the prevalence of COPD was from $11 \%-18.6 \%$ ), and is associated with higher rates of cardiovascular death, all-cause death, and aggravates outcome of any death [1012]. Arrhythmias have been associated with increased hospital admission, confers a poor prognosis, morbidity and mortality in COPD patients [4,13-15].

Large studies have shown that cardiovascular events are a leading cause of COPD-related mortality, and there is limited evidence suggesting that some of these events may be partly caused by arrhythmias [16-19]. A number literature explains the mechanism/ factors for development of arrhythmias in COPD patients secondary to adverse effects of medications like theophylline [20,21], B-agonists like salbutamol [22,23], steroids [23], anticholinergics [24], due to Cardiac autonomic dysfunction [25-28] or from ventricular failure [29]. In a health care system especially in our setting grappling with competing priorities, the importance of preemptive and early identifications of amenable conditions cannot be overemphasized which was ideally enable appropriate and cost-effective allocation of resources from complication of the disease by early screening and initiate timely management of systemic complication. One of the screenings is using simple tool like ECG to detect cardiovascular complication including arrhythmia among COPD patients. There is so far no study in our setup on the prevalence or the burden of cardiac arrhythmias among COPD patients as even ECG, simple tool is not routinely practiced. Thus, the present study, will help in establishing the burden of the problem, form a basis for further research and possibly generate recommendations on how this problem should be approached.

\section{Subjects and Methods \\ Study Design and Setting}

The study was conducted at JUMC, Jimma, one of the towns in Oromia regional state, located at South West Ethiopia, with distance of $357 \mathrm{Km}$, away from the capital city, Addis Ababa. JUMC is one of the largest teaching referral hospitals in the country, providing the health service at inpatient and outpatient level for the catchment area (more than 15 million populations) indwelling in the South West of the country. The health service is delivered by specialists, medical residents, medical interns and other health professionals. The study was conducted from May 18 to August 18, 2017 G.C among a sampled 80 COPD patients attending chest clinic of JUMC by employing a hospital based cross-sectional study design. The study populations were all COPD patients attending chest clinic of JUMC who were available during data collection period.

\section{Data Collection (Instrument, Technique)}

Dynamic pulmonary function test was carried out to diagnose and grade severity of COPD based on post bronchodilator result of forced expiratory volume in one second (FEV1) \% predicted, forced vital capacity (FVC) and (FEV1/FVC) ratio as per the guideline of COLD (1) by using dry digital spirometry (carefusion, Germany). Standard 12-lead supine resting ECG (NIHON KOHDEN Cardiofax S) was used with machine calibrated on $1 \mathrm{mV}$ for a $10 \mathrm{~mm}(0.1$ $\mathrm{mV} / \mathrm{mm}$ ) at speed of $25 \mathrm{~mm} / \mathrm{s}$, where each small box and large box represents $0.04 \mathrm{sec}$ and $0.2 \mathrm{sec}$ respectively. 10 electrodes (4 limb electrodes at right and left arms and legs +6 chest electrodes (V1V6)) were placed on client's arms, legs and chest after orientation and gel applied, yielding a total of 12 leads that measures the potential difference of movement of electrical activity of the heart.

Each ECG paper was visually analysed for recording errors, manually interpreted by investigator in liaison with the cardiologist and classified according to the Minnesota coding criteria [30], merged and thematised to different main and sub-categories for simplicity.

\section{Data Processing and Analysis}

Data was checked, categorized, coded and entered into EpiData version 3.1 after template formed and finally exported to SPSS version 20 for further analysis. Descriptive statistics like frequencies and percentages were used to describe the findings.

\section{Ethical Clearance}

Implementation of the proposal was carried out after getting approval letter from the ethical clearance committee/ethical review board of Jimma University (IRB/699/2017). An official letter of collaboration and permission request to chest and cardiac clinic of JUMC (for permission of instruments, ECG and spirometry) was obtained from Department of Physiology and Internal medicine prior to study conduction. Informed verbal and written consent were taken from the respondents/clients after explaining the objectives and purpose of the study. The participants were assured that they have full right to participate or withdraw from the study and the collected data/ information were kept confidentially. Any abnormal finding of ECG pattern (arrhythmia) observed was required consultation of physicians of chest and cardiac clinic for further interventions.

\section{Results}

\section{Baseline Characteristics of COPD Patients}

From the total sampled 80 COPD patients attending chest clinic of JUMC from May 18 to August 18, 2017 G.C, the mean age was $55.1 \pm 13.66$ that ranges from $26-90$ years by which majority of them (32.5\%) belongs to interval of 51-60 years with the ratio of males to females of $43 / 37$ (1.16:1). Majority of COPD patients (63.75\%) were experienced smoking while $60 \%$ of them especially females but also males indwelling the rural area were exposed to dusts and biomass fuel. The mean of post-bronchodilator FEV1\% predicted was (42.5 \pm 15.6$)$ as shown in detailed in Table 1 . 
Table 1: Baseline characteristics of COPD patients attending chest clinic of JUMC from May 18 to August 18, 2017 G.C, n=80.

\begin{tabular}{|c|c|}
\hline Variables & Statistics \\
\hline Age in years, mean \pm standard deviation & $55.1 \pm 13.66$ \\
\hline Sex (M/ F), Number (\%) & $43(53.8) / 37(46.25)$ \\
\hline FEV1\% predicted, mean \pm standard deviation & $42.5 \pm 15.6$ \\
\hline Smoking history (smokers/ not smokers), Number (\%) & $59(63.75) / 21(36.25)$ \\
\hline Exposure to dusts and biomass (exposed/ not), Number (\%) & $48(60.0) / 32(40.0)$ \\
\hline Duration of the disease (<5 years/ > 5years), Number (\%) & $57(71.25) / 23(28.75)$ \\
\hline Stages of COPD by GOLD (I/ II/ III/ IV), Number (\%) & $3(3.75) / 20(25.00) / 32(40.00) / 25(31.25)$ \\
\hline
\end{tabular}

\section{Prevalence of Arrhythmia among COPD Patients}

Out of the total analysed, interpreted and categorized ECG papers based on the Minnesota coding criteria from the sampled 80 COPD patients by investigator in liaison with the cardiologist, the prevalence of arrhythmia accounted about $50 \%$ or diagnosis of 40 ECG papers out of total analysed 80 ECG papers with cardiac arrhythmia. This magnitude is expected higher if detected by Holter
ECG that applied for 24 hours, but it is not available in the setup. Thus, a simple ECG that detects patients' cardiac status in a specific time was used instead with its possibility of missed diagnosis of some types of arrhythmias especially ventricular origins. The types of arrhythmia and their specific magnitude or frequency among COPD patients were analysed as follows with emphasis of possibility of presence of more than one type of arrhythmia within a single patient as detailed in Table 2 .

Table 2: Magnitude of different types of arrhythmias among COPD patients attending chest clinic of JUMC from May 18 to August 18, 2017 G.C, $n=80$.

\begin{tabular}{|c|c|c|}
\hline Types of Arrhythmia & Frequency & Percent (\%) \\
\hline Sinus origin arrhythmia (SOA) & 24 & 30.0 \\
\hline Sinus tachycardia (ST) & 7 & 8.8 \\
\hline Sinus bradycardia (SB) & 13 & 16.3 \\
\hline Sinus arrhythmia (SA) & 4 & 5.0 \\
\hline Ectopic arrhythmia (EA) & 16 & 20.0 \\
\hline Atrial flutter (Af) & 1 & 1.3 \\
\hline Atrial fibrillation $(\mathrm{AF})$ & 5 & 6.3 \\
\hline Multi focal atrial tachycardia (MAT) & 1 & 1.3 \\
\hline Premature atrial contraction (PAC) & 3 & 3.8 \\
\hline Premature ventricular contraction (PVC) & 6 & 7.5 \\
\hline Conduction block arrhythmia (CBA) & 19 & 23.8 \\
\hline Atrioventricular block (AVB) & 1 & 1.3 \\
\hline Bundle branch block (BBB) & 14 & 17.5 \\
\hline Complete right bundle branch block (CRBBB) & 3 & 3.8 \\
\hline Incomplete right bundle branch block (IRBBB) & 6 & 7.5 \\
\hline Complete left bundle branch block (CLBBB) & 4 & 5.0 \\
\hline Incomplete left bundle branch block (ILBBB) & 1 & 1.3 \\
\hline Hemi fasicular block (HFB) & 4 & 5.0 \\
\hline Left anterior hemi fasicular block (LAHFB) & 2 & 2.5 \\
\hline Left posterior hemi fasicular block (LPHFB) & 2 & 2.5 \\
\hline Other arrhythmia & 9 & 11.4 \\
\hline $\begin{array}{l}\text { [Preexcitation syndrome (PES) or Wolf Parkinson white } \\
\text { syndrome (WPWS)] }\end{array}$ & 2 & 2.5 \\
\hline Prolonged QTc interval & 7 & 8.8 \\
\hline
\end{tabular}


What have to be emphasized is, either the outcome variable (status of patient's ECG paper) was sinus normal rhythm or arrhythmic, or finally the frequency was computed and yields $50 \%$ of diagnosed cardiac arrhythmia, while left proportion might be normal sinus rhythm or can be other abnormal ECG than arrhythmia. So, the frequency was not summated with different arrhythmias, but simply the proportion of specific arrhythmia per to total analysed ECG paper.

\section{Sinus Origin Arrhythmias (SOA)}

The sinus origin arrhythmia is the most common types of arrhythmia (30\%) that detected among COPD patients. This can also be further classified to subgroups with their specific magnitude per to total analysed ECG papers, not per to occurred arrhythmias. For instance, Sinus bradycardia accounted for $16.3 \%$, Sinus tachycardia $(8.8 \%)$ and Sinus arrhythmia $(5.0 \%)$.

\section{Ectopic Arrhythmias (EA)}

Ectopic arrhythmia or non-sinus origin arrhythmia accounts $20 \%$, specifically from its subgroups the occurrence of premature ventricular Contraction was relatively highest (7.5\%), Atrial fibrillation (6.3\%), Premature atrial contraction (3.8\%), Atrial flutter $(1.3 \%)$ and Multi focal atrial tachycardia (1.3\%) among sampled COPD patients.

\section{Conduction Block Arrhythmias (CBA)}

Conduction block arrhythmia is also the second most prevalent (23.8\%) types of arrhythmia diagnosed among COPD patients, classified as Bundle branch block (17.5\%) with further division of complete bundle branch block; right $(3.8 \%)$ and left $(5 \%)$, incomplete bundle branch block; right $(7.5 \%)$ and left $(1.3 \%)$, Hemi fasicular block (5\%) and Atrioventricular block also known as heart block (1.3\%).

\section{Other Arrhythmias (PES or WPWS)}

Other arrhythmia accounts about $11.4 \%$ of arrhythmia observed among COPD patients. For instance, Preexcitation syndrome or Wolf Parkinson white syndrome and Prolonged QTc interval were discriminated by the magnitude of $2.5 \%$ and $8.8 \%$ respectively.

\section{Discussion}

The mean age of the subjects in the present study was $(55.1 \pm 13.66)$ years, while majority of the patients $(32.5 \%)$ classified at interval of 51-60 years indicates also in our setup, COPD still remains a disease of the elderly. Though, COPD was a predominantly male disease, the present study finding indicates nearly similar a male to female ratio (1.16:1). This may be not due to the low prevalence of cigarette smoking among our female population but additional burden of females' exposure to biomass fuel. The prevalence of cardiac arrhythmia in the present study was $50 \%$ which was highest and in line with studies of Hudson et al. [15] who reported the prevalence of cardiac arrhythmia 47\%. The finding of Warnier et al. [31] shows nearly similar magnitude of different types of arrhythmia with the present finding. Because both study used the same tool (Minnesota coding criteria) for classification of arrhythmia. But the magnitude of cardiac arrhythmia reported by the study of Kleiger et al. [14] and Shih et al. [32], H Zaghla et al. [33] was higher than the present finding. This difference might be because they involved only patients with severe stage of COPD, but the present study involved COPD patients from all stages. The $50 \%$ prevalence of cardiac arrhythmia diagnosed in the present study was also against the finding of Curkendall et al. [4] with lowest prevalence of $21.1 \%$, Cazzola et al. [34] with prevalence of $15.9 \%$ due to possible sample size difference, difference in severity of the disease, difference in design method, they exclude also sinus origin arrhythmias like sinus tachycardia and bradycardia which is actually diagnosed in the present study as sinus origin arrhythmia. Though, the arrhythmogenic mechanisms involved in COPD are complex and seem to have diversity across the different types of arrhythmias, the common causes for the occurrence of arrhythmias in COPD are P-wave duration and PQ interval, oxidative stress, inflammation, hypoxia, hypercapnia, pulmonary hypertension, $\beta$-adrenergic effects of bronchodilators, diastolic dysfunction, changes in atrial size by altered respiratory physiology, increased arrhythmogenicity from no pulmonary vein foci of right atrium, autonomic dysfunction, QTc dispersion, concomitant CAD and HF $[24,35,36]$.

The specific cardiac arrhythmias are discussed for their arrhythmogenic mechanisms as follows: MAT exclusively associated with COPD, developed due to increased intracellular calcium stores that induced by hypokalemia, hypoxia, acidemia, and increased catecholamines as the principal underlying mechanism $[37,38]$. $\mathrm{AF}$ is also the most prevalent (6.3\%) in the present study among COPD patients due to prolonged and inhomogeneous propagation of depolarization of atria where the right atrial electromechanical delay is significantly prolonged and negatively correlated with FEV1 that resulted in P-pulmonale, prolonged P-wave duration and $\mathrm{PQ}$ interval as a feature of AF [39]. Oxidative stress and inflammation represent major pathogenic mechanisms in AF development and perpetuation in COPD patients and facilitate atrial remodeling [4043]. Decreased oxygenation, a feature of COPD, serve also as an initiating and perpetuating factor in $\mathrm{AF}$ through cascade of hypoxiainduced vascular endothelial growth factor (VEGF) expression which contribute to atrial structural remodeling of AF and hypoxia related indirect effect of hypercapnia for a marked and uniform increase in atrial refractoriness and a significant slowing in atrial conduction $[44,45]$.

Other mechanisms are also involved for potentially linking COPD with AF (pulmonary hypertension [46,47], $\beta$-adrenergic effects of bronchodilators [23], diastolic dysfunction [48], changes in atrial size by altered respiratory physiology [49] and increased arrhythmogenicity from nonpulmonary vein foci in the right atrium [50]. Multiple potential mechanisms for ventricular arrhythmogenesis in COPD patients are Inhaled $\beta$-adrenergic agonists decreasing ventricular refractoriness [23], Autonomic neuropathy $[27,51]$ as indexes of cardiac autonomic dysfunction (heart rate variability and heart rate turbulence) [52]. The other arrhythmogenesis mechanism of most occurred types of cardiac arrhythmia (ST (8.8\%), PVC (7.5\%) and PAC (3.8\%)) among the COPD patients are due to adverse effects methylxanthine agents 
such as theophylline and aminophylline in short and long use which are well-recognized cardiotoxicity for their depolarization effects and electrolyte depletion with increased excretion of potassium and magnesium through the urine [53] and causes significant reductions in $\mathrm{AV}$ and His-Purkinje conduction intervals, sinoatrial conduction time, corrected sinus node recovery time, shortest atrial pacing interval producing 1:1 AV conduction, for their effect of adenosine A1-receptor antagonism in high concentration [21,54].

Patients receiving high dose steroids/corticosteroid therapy had increased risk of developing AF, atrial flutter, supraventricular and ventricular arrhythmias, and also increased risk of atherosclerosis, diabetes mellitus, hypertension, left atrial enlargement and IHD [55-57]. The other arrhythmogenic mechanism of AF is due highdose corticosteroids mediated potassium efflux via a direct effect on the cell membrane and its profound peripheral vasodilatation and anaphylactic reactions [57].

\section{Acknowledgment}

I express my innerfelt gratitude to Samuel Tadesse, Elsah Tegene and Tewodros G/Mariam for their incredible support during the study. I also want to thank Sr. Fantu, Sr. Worke, Sr. Saliya and Major G. for their genuine co-operation during data collection period. At last but not least, my honoured thank go to all COPD patients who participated in the study.

\section{References}

1. Rabe KF, Hurd S, Anzueto A, Barnes PJ, Buist SA, et al. (2007) Global Strategy for the Diagnosis, Management, and Prevention of Chronic Obstructive Pulmonary Disease. Am J Respir Crit Care Med pages 176(6): 532-55.

2. Murray C, Lopez A (1996) The global burden of disease: a comprehensive assessment of mortality and disability from diseases, injuries, and risk factors in 1990 and projected to 2020. Harvard University Press.

3. Menezes AMB, Perez Padilla R, Jardim JB, Muiño A, Lopez MV, et al (2005) Chronic obstructive pulmonary disease in five Latin American cities (the Platino study): A prevalence study. Lancet pages 366(9500): 1875-81.

4. Curkendall SM, Lanes S, de Luise C, Stang MR, Jones JK, et al. (2006) Chronic obstructive pulmonary disease severity and cardiovascular outcomes. Eur J Epidemiol 21(11): 803-813.

5. Fabbri LM, Hurd SS (2003) Global Strategy for the Diagnosis, Management and Prevention of COPD: 2003 update. Eur Respir J 22(1): 1-2.

6. Feary JR, Rodrigues LC, Smith CJ, Hubbard RB, Gibson JE (2010) Prevalence of major comorbidities in subjects with COPD and incidence of myocardial infarction and stroke: a comprehensive analysis using data from primary care. Thorax 65(11): 956-962.

7. Finkelstein J, Cha E, Scharf SM (2009) Chronic obstructive pulmonary disease as an independent risk factor for cardiovascular morbidity. Int J Chron Obstruct Pulmon Dis 4: 337-349.

8. Sidney S, Sorel M, Quesenberry CP, DeLuise C, Lanes S, et al. (2005) COPD and Incident Cardiovascular Disease Hospitalizations and Mortality: Kaiser Permanente Medical Care Program. Chest 128(4): 2068-2075.

9. Sin DD, Wu L, Man SFP (2005) The Relationship Between Reduced Lung Function and Cardiovascular Mortality: A Population-Based Study and a Systematic Review of the Literature. Chest 127(6): 1952-1959.

10. Loh LC, Selvarajah N, Mohan S, Wai-Sun Choo, Ismail Omar (2011) Undiagnosed COPD in Patients with Established Cardiovascular
Diseases: Prevalence, Symptoms Profiling and Functional Status. J Pulm Respir Med 01(02): 1-5.

11. Proietti M, Laroche C, Drozd M, Vijgen J, Cozma DC, et al. (2016) Impact of chronic obstructive pulmonary disease on prognosis in atrial fibrillation: A report from the EURObservational Research Programme Pilot Survey on Atrial Fibrillation (EORP-AF) General Registry. Am Heart J 181: 83-91.

12. Sin DD (2012) Mortality in COPD: The role of comorbidities. Eur Respi J 28(6): 1-13.

13. Fuso L, Antonelli Incalzi R, Pistelli R, Muzzolon R, Valente S, et al. (1995) Predicting mortality of patients hospitalized for acutely exacerbated chronic obstructive pulmonary disease. Am J Med 98(3): 272-277.

14. Kleiger RE, Senior RM (1974) Longterm Electrocardiographic Monitoring of Ambulatory Patients with Chronic Airway Obstruction. Chest 65(5): 483-487.

15. Hudson LD, Kurt TL, Petty TL, Genton E (1973) Arrhythmias Associated with Acute Respiratory Failure in Patients with Chronic Airway Obstruction. Chest 63(5): 661-665.

16. Kusunoki Y, Nakamura T, Hattori K, Motegi T, Ishii T, et al. (2016) Atrial and Ventricular Arrhythmia-Associated Factors in Stable Patients with Chronic Obstructive Pulmonary Disease. Respiration 91(1): 34-42.

17. Friedman GD, Klatsky AL, Siegelaub AB (1976) Lung Function and Risk of Myocardial Infarction and Sudden Cardiac Death. N Engl J Med 294(20): 1071-1075.

18. Baillard C, Boussarsar M, Fosse JP, Girou E, Le Toumelin P, et al. (2003) Cardiac troponin I in patients with severe exacerbation of chronic obstructive pulmonary disease. Intensive Care Med 29(4): 584-589.

19. Agustí AGN, Noguera A, Sauleda J, Sala E, Pons J, et al. (2003) Systemic effects of chronic obstructive pulmonary disease. Eur Respir J 21(2): 347-360.

20. Bittar G, Friedman HS (1991) The Arrhythmogenicity of Theophylline: A Multivariate Analysis of Clinical Determinants. Chest 99(6): 1415-1420.

21. Sessler CN, Cohen MD (1990) Cardiac Arrhythmias during Theophylline Toxicity: A Prospective Continuous Electrocardiographic Study. Chest 98(3): 672-678.

22. Hanrahan JP, Grogan DR, Baumgartner RA, Wilson A, Cheng H, et al. (2008) Arrhythmias in patients with Chronic Obstructive Pulmonary Disease (COPD): Occurrence frequency and the effect of treatment with the inhaled long-acting beta2-agonists arformoterol and salmeterol. Medicine (Baltimore) 87(6): 319-328.

23. Kallergis EM, Manios EG, Kanoupakis EM, Schiza SE, Mavrakis HE, et al. (2005) Acute Electrophysiologic Effects of Inhaled Salbutamol in Humans. Chest 127(6): 2057-2063.

24. Goudis CA, Konstantinidis AK, Ntalas IV, Korantzopoulos P (2015) Electrocardiographic abnormalities and cardiac arrhythmias in chronic obstructive pulmonary disease. Int J Cardiol 199: 264-273.

25. Tükek T, Yildiz P, Akkaya V, Akif Karan M, Atilgan D, et al. (2002) Factors Associated with the Development of Atrial Fibrillation in COPD Patients: The Role of P-Wave Dispersion. Ann Noninvasive Electrocardiol 7(3): 222-227.

26. Yildiz P, Tükek T, Akkaya V, Sözen AB, Yildiz A, et al. (2002) Ventricular Arrhythmias in Patients with COPD Are Associated with QT Dispersion. Chest 122(6): 2055-2061.

27. Stewart AG, Waterhouse JC, Howard P (1995) The QTc interval, autonomic neuropathy and mortality in hypoxaemic COPD. Respir Med 89(2): 79-84.

28. Tükek T, Yildiz P, Atilgan D, Tuzcu V, Eren M, et al. (2003) Effect of diurnal variability of heart rate on development of arrhythmia in patients with chronic obstructive pulmonary disease. Int J Cardiol 88(2-3): 199-206. 
29. Incalzi RA, Pistelli R, Fuso L, Cocchi A, Bonetti MG, et al. (1990) Cardiac Arrhythmias and Left Ventricular Function in Respiratory Failure from Chronic Obstructive Pulmonary Disease. Chest 97(5): 1092-1097.

30. Rautaharju PM, Calhoun HP, Chaitman BR (1991) NOVACODE serial ECG classification system for clinical trials and epidemiologic studies. J Electrocardiol pages 24: 179-187.

31. Warnier MJ, Rutten FH, Numans ME, Kors JA, Tan HL, et al. (2013) Electrocardiographic characteristics of patients with chronic obstructive pulmonary disease. COPD J Chronic Obstr Pulm Dis 10(1): 62-71.

32. Shih HT, Webb CR, Conway WA, Peterson E, Tilley B, et al. (1988) Frequency and Significance of Cardiac Arrhythmias in Chronic Obstructive Lung Disease. Chest 94(1): 44-48.

33. Zaghla H, Al Atroush H, Samir A, Kamal M (2013) Arrhythmias in patients with chronic obstructive pulmonary disease. Egypt J Chest Dis Tuberc 62(3): 377-385.

34. Cazzola M, Bettoncelli G, Sessa E, Cricelli C, Biscione G (2010) Prevalence of comorbidities in patients with chronic obstructive pulmonary disease. Respiration 80(2): 112-119.

35. Bhatt SP, Dransfield MT (2013) Chronic obstructive pulmonary disease and cardiovascular disease. Transl Res 162(4): 237-251.

36. Goudis CA (2017) Chronic obstructive pulmonary disease and atrial fibrillation: An unknown relationship. J Cardiol 69(5): 699-705.

37. Scher D, Arsura EL (1989) Multifocal atrial tachycardia: mechanisms, clinical correlates, and treatment. Am Hear J 118(3): 574-580.

38. McCord J, Borzak S (1998) Multifocal Atrial Tachycardia. Chest 113(1): 203-209.

39. Caglar IM, Dasli T, Turhan Caglar FN, Teber MK, Ugurlucan M, et al. (2012) Evaluation of atrial conduction features with tissue doppler imaging in patients with chronic obstructive pulmonary disease. Clin Res Cardiol 101(8): 599-606.

40. Korantzopoulos P, Kolettis TM, Galaris D, Goudevenos JA (2007) The role of oxidative stress in the pathogenesis and perpetuation of atrial fibrillation. Int J Cardiol 115(2): 135-143.

41. Gan WQ Man SFP, Senthilselvan A, Sin DD (2004) Association between chronic obstructive pulmonary disease and systemic inflammation: a systematic review and a meta-analysis. Thorax 59(7): 574-580.

42. Kirkham PA, Barnes PJ (2013) Oxidative Stress in COPD. Chest 144(1): 266-273.

43. Guo Y, Lip GYH, Apostolakis S (2012) Inflammation in Atrial Fibrillation. J Am Coll Cardiol 60(22): 2263-2270.

ISSN: 2574-1241

DOI: $10.26717 / B J S T R .2018 .10 .002001$

Wondu Reta Demissie. Biomed J Sci \& Tech Res

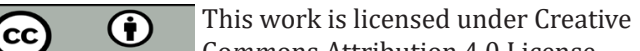

This work is licensed under Creative

Submission Link: https://biomedres.us/submit-manuscript.php
44. Ogi H, Nakano Y, Niida S, Dote K, Y Hirai, et al. (2010) Is structural remodeling of fibrillated atria the consequence of tissue hypoxia? Circ J 74(9): 1815-1821.

45. Stevenson IH, Roberts Thomson KC, Kistler PM, Edwards GA, Spence S, et al. (2010) Atrial electrophysiology is altered by acute hypercapnia but not hypoxemia: Implications for promotion of atrial fibrillation in pulmonary disease and sleep apnea. Hear Rhythm 7(9): 1263-1270.

46. Buch P, Friberg J, Scharling H, Lange P, Prescott E (2003) Reduced lung function and risk of atrial fibrillation in the Copenhagen City Heart Study. Eur Respir J 21(6): 1012-1016.

47. Shibata Y, Watanabe T, Osaka D, Abe S, Inoue S, et al. (2011) Impairment of pulmonary function is an independent risk factor for atrial fibrillation: the Takahata study. Int J Med Sci 8(7): 514-522.

48. Caram L, Ferrari R, Naves C, Tanni S, Coelho L, et al. (2013) Association between left ventricular diastolic dysfunction and severity of chronic obstructive pulmonary disease. Clinics 68(6): 772-776.

49. Koshino Y, Villarraga HR, Orban M, Bruce CJ, Pressman GS, et al. (2010) Changes in Left and Right Ventricular Mechanics During the Mueller Maneuver in Healthy Adults. Circ Cardiovasc Imaging 3(3): 282-289.

50. Roh SY, Choi JI, Lee JY, Kwak JJ, Park JS, et al. (2011) Catheter Ablation of Atrial Fibrillation in Patients with Chronic Lung Disease. Circ Arrhythmia Electrophysiol 4(6): 815-822.

51. Chhabra SK, De S (2005) Cardiovascular autonomic neuropathy in chronic obstructive pulmonary disease. Respir Med 99(1): 126-133.

52. Gunduz H, Talay F, Arinc H, Ozyildirim S, Akdemir R, et al. (2009) Heart rate variability and heart rate turbulence in patients with chronic obstructive pulmonary disease. Cardiology journal 16(6): 553-559.

53. Wood Baker R, Cochrane B, Naughton MT (2010) Cardiovascular mortality and morbidity in chronic obstructive pulmonary disease: the impact of bronchodilator treatment. Intern Med J 40(2): 94-101.

54. Barnes PJ (2013) Theophylline. Am J Respir Crit Care Med 188(8): 901906.

55. Huerta C, Lanes SF, García Rodríguez LA (2005) Respiratory medications and the risk of cardiac arrhythmias. Epidemiology 16(3): 360-366.

56. Christiansen CF, Christensen S, Mehnert F, Cummings SR, Chapurlat RD, et al. (2009) Glucocorticoid Use and Risk of Atrial Fibrillation or Flutter. Arch Intern Med 169(18): 1677-1683.

57. van der Hooft CS, Heeringa J, Brusselle GG, Hofman A, Witteman JCM, et al. (2006) Corticosteroids and the Risk of Atrial Fibrillation. Arch Intern Med 166(9): 1016-1020.

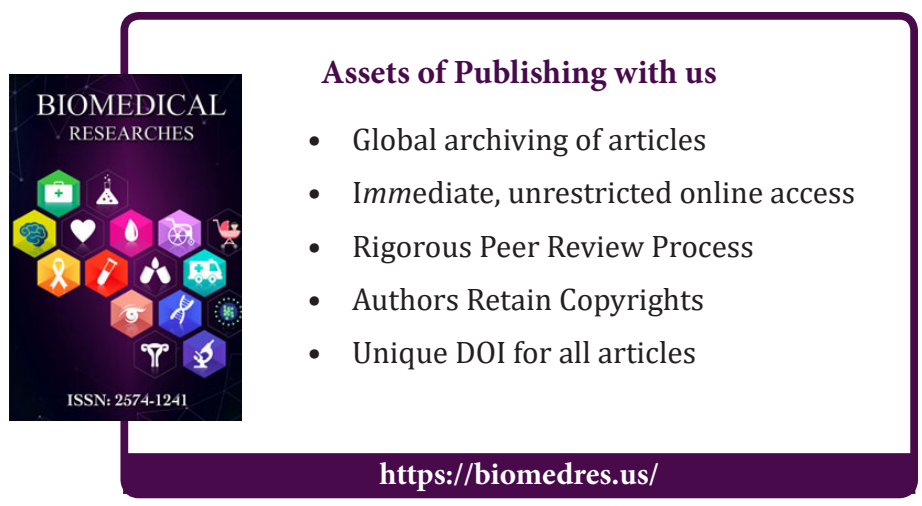

Cite this article: Wondu Reta Demissie. Prevalence of Cardiac Arrhythmias among Chronic Obstructive Pulmonary Disease Patients Admitted to Jimma University Medical Center. Biomed J Sci\&Tech Res 10(5)-2018. BJSTR. MS.ID.002001. D0I: 10.26717/ BJSTR.2018.10.002001. 